Draft version November 10, 2009

Preprint typeset using $\mathrm{LAT}_{\mathrm{E}} \mathrm{X}$ style emulateapj v. 08/22/09

\title{
THE TEMPERATURE OF THE COSMIC MICROWAVE BACKGROUND
}

\author{
D. J. FIXSEN ${ }^{1}$ \\ Draft version November 10, 2009
}

\begin{abstract}
The FIRAS data are independently recalibrated using the WMAP data to obtain a CMB temperature of $2.7260 \pm 0.0013$. Measurements of the temperature of the cosmic microwave background are reviewed. The determination from the measurements from the literature is cosmic microwave background temperature of $2.72548 \pm 0.00057 \mathrm{~K}$.
\end{abstract}

Subject headings: cosmology: microwave background - cosmology: observations

\section{INTRODUCTION}

The Wilkinson Microwave Anisotropy Probe (WMAP) data presents an opportunity to recalibrate the Far InfraRed Absolute Spectrophotometer (FIRAS) experiment and produce an independent check of the other measurements of the cosmic microwave background (CMB) temperature. In sections $2 \& 3$ the WMAP data will be presented and combined with the FIRAS data to make an independent estimate of the CMB temperature. In sections 4 \& 5 this new estimate will be combined with others from the literature to generate an improved estimate for the CMB temperature.

The WMAP data only measures the difference in intensity between different points on the sky. However, the precision is sufficient such that the velocity of the WMAP spacecraft can be used to calibrate the velocity to various points on the surface of last scattering of the CMB. This velocity in turn is used to form a differential spectrum of the CMB. The differential spectrum is then fit with a single parameter which is the CMB temperature.

\section{WMAP VELOCITY MAP}

The standard WMAP sky maps (Hinshaw et al. 2009) are corrected to the baricenter of the solar system using the JPL ephemeris (Standish \& Fienga 2002). The calibration assumes a CMB temperature of $2.725 \mathrm{~K}$ (derived from the FIRAS measurement). However, the various changes as the WMAP makes its way around the sun (now in its ninth repetition) can be used to calibrate the WMAP data in terms of velocity. The velocity of the WMAP spacecraft, with respect to the sun, is known to $<1 \mathrm{~cm} / \mathrm{s}$, which is a negligible uncertainty compared to other uncertainties considered here. This velocity is used to calibrate a map of velocity relative to the surface of last scattering of the CMB. Most of this velocity is the dipole, presumably the motion of the solar system with respect to the frame of the CMB, however it includes temperature variations due to the Sachs-Wolfe effect which has the same spectrum. This process is repeated for each WMAP differential assembly and each year yielding 50 independent maps for the first 5 years of WMAP operation.

The WMAP velocity maps are the temperature maps, available at http://lambda.gsfc.nasa.gov/

\footnotetext{
${ }^{1}$ University of Maryland, Goddard Space Flight Center
}

product/map/current/m_products.cfm, with the dipole added back in and divided by the CMB temperature. In generating the WMAP temperature maps a dipole is fit and removed from the raw data. The residual variations are due to various sensitivities of the WMAP instrument, the changing velocity as the spacecraft makes its annual trek around the solar system and the small variations of the CMB as a function of position. The WMAP team has done an excellent job of removing the instrumental effects. By correcting the velocity to the barycenter of the solar system, the effects of the spacecraft velocity are also removed. But this information also allows the calibration of the WMAP data. But the information is in velocity rather than temperature. In order to get the temperature for the published maps an absolute temperature must be assumed. The WMAP team used $2.725 \mathrm{~K}$ from the previous FIRAS measurements to translate the velocity measurements into temperature units. To get the velocity maps, the data is divided by $2.725 \mathrm{~K}$ to restore the velocity calibration in units of $v / c$. The dipole $\left(v / c=0.0012338, l=263.87^{\circ}, b=48.24^{\circ}\right)$ is added back in to restore the full velocity. The fitting includes an absolute term which is treated as a nuisance parameter so no absolute adjustment to the WMAP data is required or made.

No corrections to the WMAP data are made for Galactic foregrounds. The maps are convolved with the FIRAS beam (approximately a $7 \mathrm{deg}$ tophat) to produce maps with the FIRAS resolution. These maps are produced with the pixelization of the native FIRAS data.

For each velocity map, the FIRAS data (also available on the lambda website) are fit to a set of ten templates, shown in figure 1. The first template is unity everywhere. This template is included to model the monopole. The second template is one of the velocity maps from the WMAP data. The remaining eight maps are included to fit various foregrounds. These templates include the FIRAS CII and NII maps (Fixsen et al. 1999). The Band 8 and 10 DIRBE data also from the COBE mission and a model of the zodiacal emission from the DIRBE team (Kelsall et al. 1998). Also included are an Hi map (Dickey \& Lockman 1990, Hartmann \& Burton 1997), an Aluminum-26 emission line map (Diehl et al. 1995) and the Haslam $408 \mathrm{MHz}$ map (Haslam et al. 1981). The maps not derived from FIRAS data are convolved with the FIRAS beam. Templates 3-10 are included to 

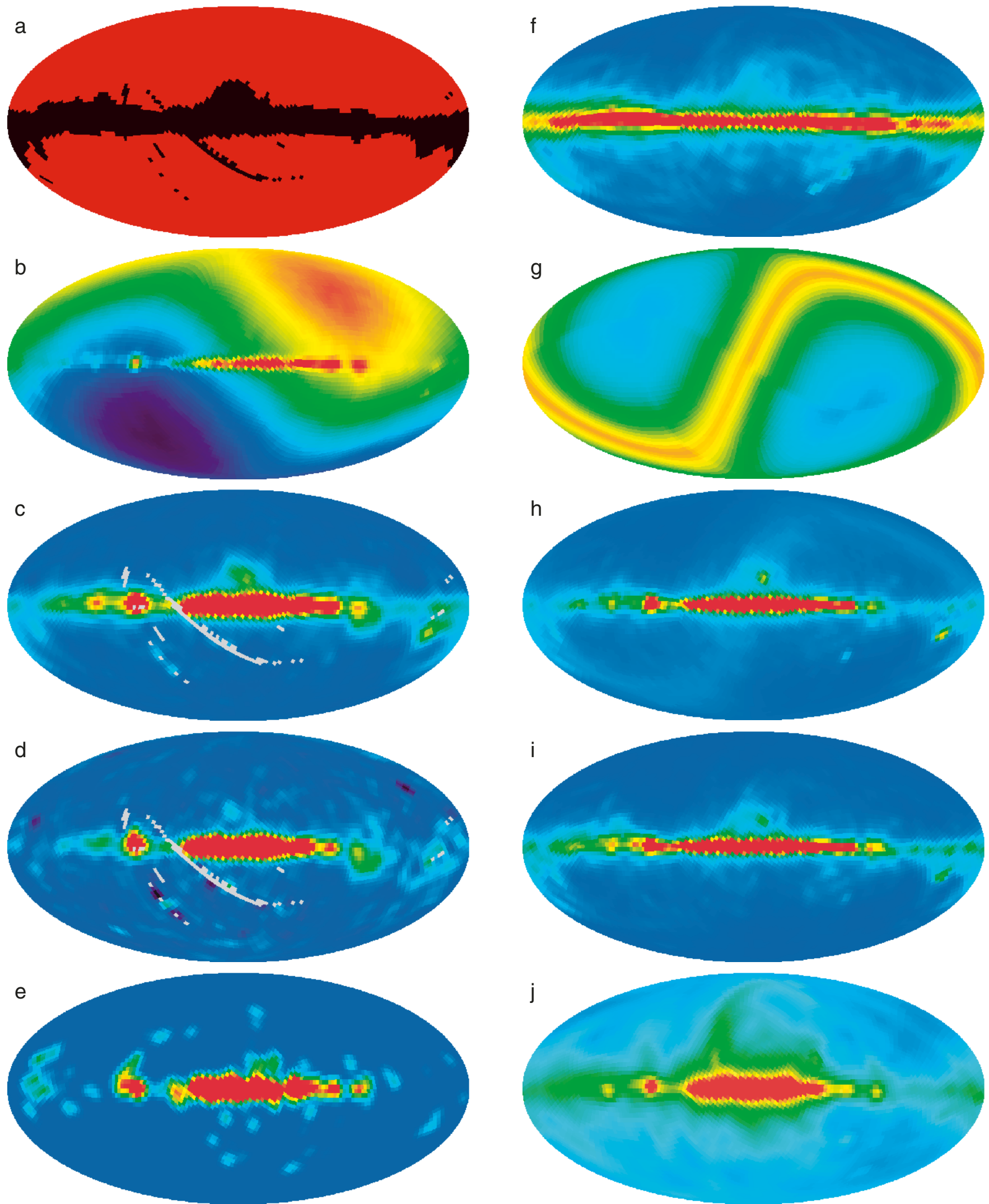

FIG. 1. - The templates used to fit the FIRAS data. The templates are in Galactic coordinates with the center of the Galaxy in the center of each Mollwide projection. The Galactic equator is a horizontal line across the middle of each figure. From top to bottom the templates are a)Data mask b)WMAP velocity c)CiI d)NII e)Al26 f)Hi g)Zodical model h)DIRBE band 8 i)DIRBE band 10 j)Haslam 408 $\mathrm{MHz}$. 


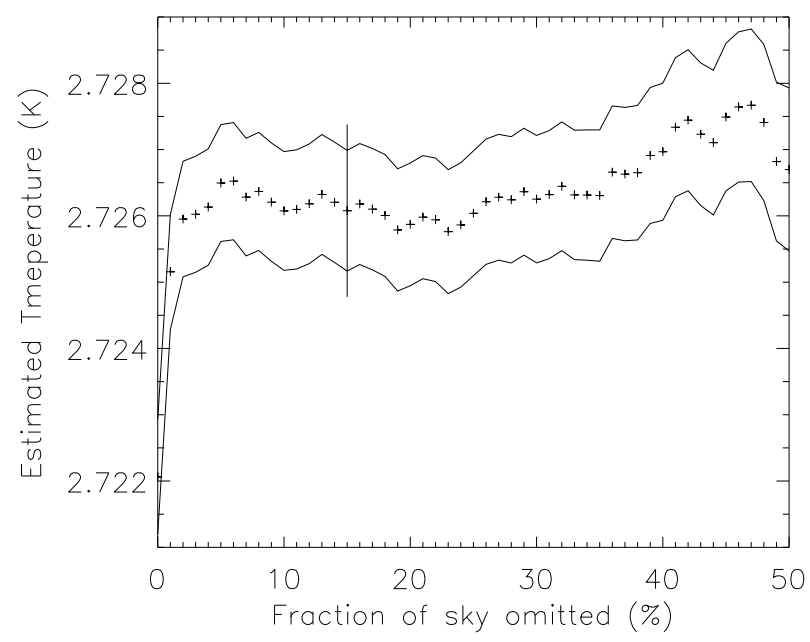

FIG. 2.- The crosses are the CMB temperature estimation for a given fraction of the brightest part of the sky excluded. The lines are the nominal $+1 \sigma$ and $-1 \sigma$ limits. The error bar is the adopted value and uncertainty inflated for the excess $\chi^{2}$.

attempt to fit all local features even though many subsets of them would do almost as well. Since there are 6063 pixels the 8 templates make an insignificant reduction in the total number of degrees of freedom.

The FIRAS data is arbitrarily cut off at $1 \mathrm{THz}$ since the CMB spectrum is essentially zero beyond that frequency and the uncertainty of the FIRAS data at high frequencies is not important for this analysis. The fits use the weight map of the FIRAS low frequency data (which is nearly identical in form to the high frequency weight map). Each frequency is fit separately and so each template generates a spectrum for each fit. All of the spectra except the spectrum associated with the velocity are treated as nuisance parameters. These increase the uncertainty but are otherwise unused.

Even with these templates the Galactic plane is too large a perturbation to be insignificant. So the pixels with the largest DIRBE band 10 signal are excluded from the fit. The process was tested with $0 \%$ to $90 \%$ of the data excluded. Figure 2 shows the average estimated temperature for all $50 \mathrm{WMAP}$ channels and years as a function of the fraction of excluded data. For less than $5 \%$ of the data excluded, significant variations are seen. The variations are concentrated in the the $22 \mathrm{GHz}(\mathrm{K}$ band) templates. If more than $50 \%$ of the data are excluded the uncertainties are significantly larger, not only due to the data loss but also due to the loss of the hot and cold ends of the CMB dipole. The results that follow are for $15 \%$ of the data excluded. These fits, done independently at each frequency, result in 50 spectra.

\section{CMB VELOCITY TEMPERATURE}

The average of the 50 spectra is shown in fig. 3. Since this spectrum is associated with the velocity of the solar system with respect to the CMB (and some SachsWolfe effect), the spectrum appears not as an absolute spectrum but as a differential spectrum generated by the doppler shift.

According to the special theory of relativity the spectrum observed in a reference frame moving with respect to the source of a black-body spectrum, $B(T, \nu)$, at tem- perature $T$, is shifted such that

$$
S^{\prime}(\nu)=B\left(T \sqrt{\frac{1+v}{1-v}}, \nu\right)
$$

where $\nu$ is the frequency and $v$ is the velocity towards the source divided by the speed of light.

Thus ignoring negligible second order terms and higher we have:

$$
S(\nu, p)=B(T, \nu)+\left.v(p) T \frac{\partial B(T, \nu)}{\partial T}\right|_{T=T_{0}}
$$

where the spectrum is now a function of position, $P$, on the sky. Note that the first term is absorbed in the first template. The remaining term, $v T \partial B / \partial T$ is the term related to the 2 nd template. The velocity, $v$, is already included in the template, thus the term to fit is $T \partial B / \partial T$, which must match the spectrum. Although strictly speaking this is nonlinear, a spectrum $T \partial B / \partial T$ for a $T$ near the CMB temperature (say $2.726 \mathrm{~K}$ ) can be subtracted from the spectrum and the residual can then be fit to $\delta T\left(\partial B / \partial T+T \partial^{2} B / \partial T^{2}\right)$ for the small $\delta T$ correction. Since both the process of averaging the velocity maps and the spectral fitting are linear processes, they can be done in either order. So the average spectrum is fit with a result of $T=2.7260$.

\subsection{FIRAS Uncertainty}

The uncertainty of the temperature is dominated by the noise in the FIRAS measurements. Propagating the uncertainties shown in figure 3 results in an uncertainty estimate of $0.74 \mathrm{mK}$, but this does not include the correlations amongst the different frequencies. Including the correlations and the PEP error term, which is important in this context (Fixsen et al. 1994), results in an uncertainty estimate of $1.09 \mathrm{mK}$. The $\chi^{2}$ is 98.7 for $69 \mathrm{DOF}$. Since the $\chi^{2}$ is higher than expected the uncertainty is inflated to produce a $\chi^{2}$ per DOF of unity with a resulting uncertainty of $1.3 \mathrm{mK}$ in the CMB temperature due to the uncertainties of the FIRAS measurements.

In deriving the FIRAS dipole (Fixsen et al. 1996) amplitude the only term is the $\delta T \partial B / \partial T$. Here the second term $\delta T T \partial^{2} B / \partial T^{2}$ is both larger and at a mean higher frequency. This allows a more precise determination of the temperature. Also the velocity map allows more control of the systematic effects from the cut. Some of the variation due to the cuts was due to higher order $(l>1)$ variation in the CMB. Here the variation in the $\mathrm{CMB}$ is included in the velocity maps, so neither of these terms add to the uncertainty.

\subsection{WMAP Uncertainty}

The uncertainty from the WMAP measurements also needs to be included. The RMS variation in the gains $(\Delta v / v)$ of the 50 independent WMAP maps is 0.00096 . Nominally, the uncertainty of the mean of such a set would be 0.00014 . Figure 7 of Hinshaw et al. (2009) indicates a .001 uncertainty for each of 40 individually calibrated data channels which results in a similar uncertainty estimate for the mean.

But at the level of 0.00014, the WMAP uncertainties are insignificant. Even if the uncertainty were 0.0005 the WMAP data would not the be a significant source of 


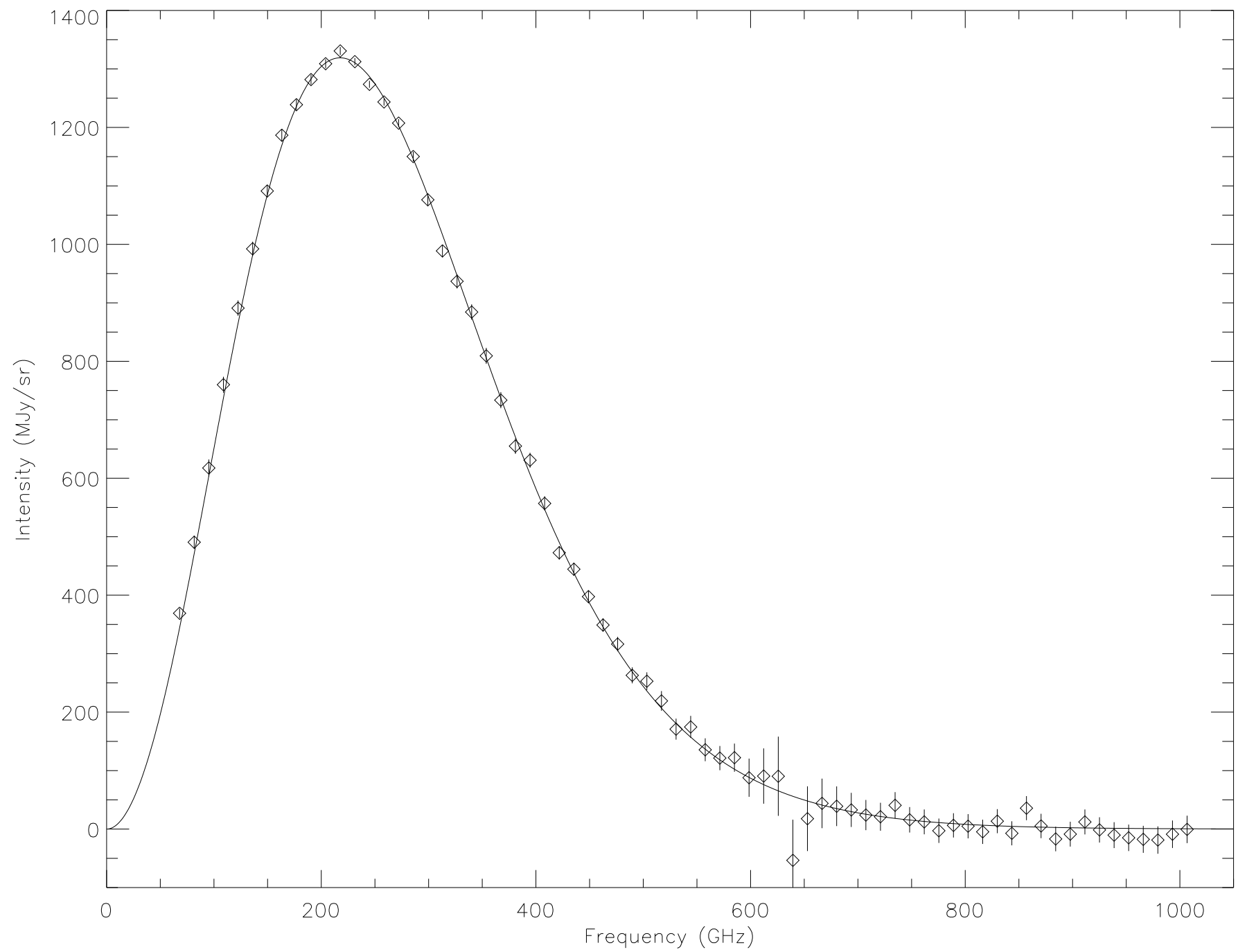

FIG. 3.- The mean spectrum associated with the velocity of the solar system with respect to the CMB. The line is the $a$ priori prediction based on the WMAP velocity and the previous FIRAS calibration. The uncertainties are the noise from the FIRAS measurements. The error bars are slightly misleading, because they do not show the correlations, but the correlated errors are properly treated in the fit.

uncertainty. If the mean error were 0.0016, the WMAP uncertainty would equal the uncertainty from the FIRAS uncertainty and the final uncertainty would be increased by a factor of $\sqrt{2}$. Each WMAP channel and year is processed independently. Unless there is a serious unexplained error in the WMAP data that correlates both channels and years the uncertainty of the WMAP data is insignificant relative to the uncertainty in the FIRAS data.

The fitted temperatures for each WMAP channel and year is shown in table 1 . The average is $2.7260 \mathrm{~K}$ with a standard deviation of $0.6 \mathrm{mK}$. The implied uncertainty of the mean is $0.09 \mathrm{mK}$. The standard deviation is smaller than expected because a significant part of the variation of the WMAP velocity maps is concentrated in the Galactic plane. The $0.09 \mathrm{mK}$ does not include the common uncertainty of the WMAP velocity maps or the FIRAS errors.

Combining the uncertainties of the FIRAS and WMAP in quadrature results in an uncertainty of $1.3 \mathrm{mK}$. This measurement is unaffected by absolute systematic errors of either FIRAS or WMAP as it uses only

\begin{tabular}{rccccc} 
WMAP & Year 1 & Year 2 & Year 3 & Year 4 & Year 5 \\
\hline K & 2.72511 & 2.72517 & 2.72522 & 2.72512 & 2.72519 \\
Ka & 2.72532 & 2.72530 & 2.72526 & 2.72529 & 2.72544 \\
Q1 & 2.72555 & 2.72541 & 2.72540 & 2.72546 & 2.72557 \\
Q2 & 2.72553 & 2.72548 & 2.72538 & 2.72543 & 2.72544 \\
V1 & 2.72625 & 2.72627 & 2.72621 & 2.72620 & 2.72639 \\
V2 & 2.72653 & 2.72622 & 2.72630 & 2.72626 & 2.72662 \\
W1 & 2.72624 & 2.72616 & 2.72644 & 2.72635 & 2.72634 \\
W2 & 2.72681 & 2.72682 & 2.72656 & 2.72665 & 2.72650 \\
W3 & 2.72675 & 2.72678 & 2.72649 & 2.72686 & 2.72714 \\
W4 & 2.72712 & 2.72685 & 2.72617 & 2.72580 & 2.72658
\end{tabular}

TABLE 1

ESTIMATED CMB TEMPERATURES FOR THE VARIOUS YEARS AND WMAP CHANNELS. ALL OF THESE FITS ARE WITH THE BRIGHTEST $15 \%$ OF THE SKY EXCLUDED.

differential measurements of both experiments. Further it is insensitive to long term offset drifts in either instrument as the measurement is dominated by the measurement at the precession period of the WMAP data (about 60 minutes) and the orbital period of the COBE spacecraft (about 100 minutes). 
In particular it does not depend on the absolute calibration of the FIRAS thermometers although it does depend on the gain calibration of the FIRAS external calibrator thermometers. It also is relatively insensitive to the FIRAS frequency calibration as the measurement uncertainty is dominated by the amplitude of the spectrum.

The diamonds in fig 3 are the spectra derived from the FIRAS data. The uncertainties shown are also derived from the FIRAS data. Note the line in figure 3 is not a fit but the predicted line from the previous $\mathrm{T}=2.725$ FIRAS calibration. The best fit is $2.7260 \pm 0.0013 \mathrm{~K}$. The feature at $\nu=630 \mathrm{GHz}$, including the larger uncertainty, is due to the dichroic filter separating the low and high frequencies in the FIRAS instrument.

\section{CMB TEMPERATURE}

There were many publications of measurements of the CMB temperature from the late '60s and '70s. But the uncertainties are large and the systematics were not well understood. Here an arbitrary cutoff of $50 \mathrm{mK}$ uncertainty was used to select 15 sources for the CMB temperature from recent publications. These results are shown in Table 2.

\begin{tabular}{|c|c|c|c|}
\hline $\begin{array}{l}\text { CMB } \\
\text { Source }\end{array}$ & $\begin{array}{l}\text { Temp } \\
(\mathrm{K})\end{array}$ & $\begin{array}{c}\text { Uncertainty } \\
(\mathrm{mK})\end{array}$ & Reference \\
\hline $\mathrm{CN}$ & 2.700 & 40 & Meyer \& Jura (1985) \\
\hline $\mathrm{CN}$ & 2.740 & 50 & Crane et al. (1986) \\
\hline Balloon & 2.783 & 25 & Johnson \& Wilkinson (1987) \\
\hline $\mathrm{CN}$ & 2.750 & 40 & Kaiser \& Wright (1990 \\
\hline Rocket & 2.736 & 17 & Gush et al. (1990) \\
\hline S Pole & 2.640 & 39 & Levin et al. (1992) \\
\hline Balloon & 2.712 & 20 & Schuster et al. (1993) \\
\hline $\mathrm{CN}$ & 2.796 & 39 & Crane et al. (1994) \\
\hline $\mathrm{CN}$ & 2.729 & 31 & Roth et al. (1995) \\
\hline Balloon & 2.730 & 14 & Staggs et al. (1996) \\
\hline ARCADE1 & 2.694 & 32 & Fixsen et al. (2004) \\
\hline ARCADE1 & 2.721 & 10 & Fixsen et al. (2004) \\
\hline ARCADE2 & 2.731 & 5 & Fixsen et al. (2009) \\
\hline FIRAS & 2.7249 & 1.0 & Mather et al. (1999) \\
\hline FIRAS & 2.7255 & 0.85 & Fixsen et al. (1996) \\
\hline FIRAS & 2.7260 & 1.3 & This Work \\
\hline Mean & 2.72548 & .57 & \\
\hline
\end{tabular}

TABLE 2

Measurements and uncertainties of the CMB TEMPERATURE.

The weight of the measurements is dominated by the FIRAS measurements, but it is still instructive to look at the other measurements. The measurements using $\mathrm{CN}$ are entirely different from any of the other measurements. But the combined CN measurements are $2.742 \pm 0.017 \mathrm{~K}$ which is only one sigma high. The rocket measurement from Gush, Halpern and Wishnow (1990) is like the FIRAS measurement in that it uses a fourier transform spectrometer, but it has an independent calibrator and independent thermometers. The other measurements depend on external calibrators as the absolute reference, but these are comparatively narrow bands on the low frequency side of peak of the $\mathrm{CMB}$ radiation. The $\chi^{2}$ for the 16 measurements is 17.9 for 15 DOF. A $\chi^{2}$ this large should be expected about $26 \%$ of the time. Most of the excess $\chi^{2}$ comes from three measurements: the Johnson \& Wilkinson balloon measurement, the South Pole measurement, and the fourth $\mathrm{CN}$ measurement. With this number of measurements, one or two $2 \sigma$ results should be expected but here there are three.

Without the FIRAS measurements the weighted average is $2.729 \pm 0.0038$ which is $1.0 \sigma$ from the final answer. Most of the weight (97\%)of the final temperature estimate is from the FIRAS measurements. Each FIRAS measurement will be reviewed in turn.

The original concept of the FIRAS instrument was that the sky would be observed and the internal reference would be adjusted to minimize the signal. Then the external calibrator would be inserted and adjusted to match the signal from the sky. This method depends on knowing the calibration of the external calibrator germanium resistance thermometers. A $5 \mathrm{mK}$ error in the original temperature determination of the external calibrator led directly to a $5 \mathrm{mK}$ error in the temperature determination of the CMB. However, the calibration process corrects other effects of the error to first order (Fixsen et al. 1994). There were slight modifications to this plan (eg. the internal reference was offset $10 \mathrm{mK}$ for about half of the data), but basically the result is $2.7249 \mathrm{~K}$ (This has been rounded off to 2.725 in the literature), including the $5 \mathrm{mK}$ correction for thermometer self-heating in the high current mode. The low current mode is noisy but there are $\sim 10^{5}$ measurements to compare the low current readings to the high current readings all at $\sim 2.7 \mathrm{~K}$. The final uncertainty depends on the calibration of the thermometers at NIST and the readout electronics. The uncertainty is estimated as $1 \mathrm{mK}$.

The second calibration of the FIRAS data is based on the color. The temperature can be determined from the color of the radiation if the frequency scale can be accurately determined. The frequency scale is derived from FIRAS observations of the interstellar CO and [C I] lines at 1300, 867, 650, and $609 \mu \mathrm{m}$ (Fixsen et al. 1996). These were chosen because they are bright enough to determine the frequency and they are in the same part of the spectrum as the CMB. The temperature scale was determined independently from 7 different combinations of the four detectors and three scan modes. These determinations agreed within their uncertainties and the weighted uncertainty is $0.2 \mathrm{mK}$. There is a common uncertainty of $0.82 \mathrm{mK}$ due to the uncertainty of the frequency scale, which dominates the total uncertainty. The result is $2.7255 \mathrm{~K} \pm 0.85 \mathrm{mK}$. The thermometer errors are only weakly coupled to the color temperatures. Indeed, when the first color temperature was published, it disagreed with the thermometer by $4.5 \sigma$. It was only with the discovery of the high current self-heating offset that the two measurements came into alignment. The uncertainty of this method is driven by the uncertainty in the measurement of the frequency of the $\mathrm{CO}$ and $[\mathrm{C} \mathrm{I}]$ lines.

Now there is a third independent method of precisely calibrating the FIRAS instrument. The velocity method was presented before with the COBE DMR data (Fixsen et al. 1996). However, the DMR data did not have sufficient velocity precision to fully exploit this method; the WMAP data do. Because the differential measurements from the FIRAS instrument are taken only 50 minutes (half of an orbit) apart with the instrument in substan- 
tially the same state this method has the least potential for systematic errors. These spectra have far more supporting data than the calibration data. Each of the WMAP frequencies can be used to construct velocity map which in turn can be used to construct a spectrum. These spectra can then be fit to a dB/dT spectrum with the temperature as the single adjustable parameter.

\section{SUMMARY AND CONCLUSIONS}

The calibration methods for the Far Infrared Absolute Spectrophotometer (FIRAS) have been described and the accuracy estimated. All of the recent precision estimates of the CMB temperature agree within 2.5 times their uncertainties. These estimates were made with a variety of methods from different platforms and different frequencies. Combining all of the estimates results in a very modestly elevated $\chi^{2}$ and an improved absolute temperature estimation of $2.72548 \pm 0.00057 \mathrm{~K}$.

I thank the WMAP team for providing the smoothed sky maps in velocity units. A special thanks to J Weiland and G Hinshaw.

\section{REFERENCES}

Crane P et al. , 1986, ApJ, 309, 882

Crane P et al. , 1994, ApJ, 346, 136

Dickey JM \& Lockman FJ, 1990, Annual review of astronomy and astrophysics, 28, 215

Diehl R et al. , 1995, A\&A, 298, 445

Fixsen DJ et al. , 1994a, ApJ, 420, 457

Fixsen DJ et al. , 1996, ApJ, 473, 576

Fixsen DJ et al. , 1999, ApJ, 526, 207

Fixsen DJ et al. , 2004, ApJ, 612, 86

Fixsen DJ et al. , 2009, ApJ, in press

Gush HP, Halpern M \& Wishnow E, 1990, Phys Rev Lett, 65, 537

Hartmann D \& Burton BW, "Atlas of Galactic Neutral Hydrogen", pp. 243. ISBN 0521471117. Cambridge, UK: Cambridge University Press, 1997.
Haslam CGT et al. 1981, A\&A, 100, 209

Hinshaw G et al. , 2009, ApJS, 180, 225

Johnson D \& Wilkinson D, 1987, ApJ, 313, L1

Kaiser ME \& Wright EL 1990, ApJ, 356, L1

Kelsall T et al. , 1998, ApJ, 508, 44

Levin S et al. , 1992, ApJ, 396, 3

Mather JC et al. , 1999, ApJ, 512, 511

Meyer DM \& Jura M 1985, ApJ, 297, 119

Roth KC et al. , 1995, ApJ, 420, 457

Schuster J et al. , 1993, ApJ412, 471993

Staggs S et al. , 1996, ApJ, 473, L1

Standish EM \& Fienga A 2002, A\&A, 384, 322 